\title{
Non-Local Interactions and the Excitation Spectrum in Lattice Quantum Scalar Field Models*
}

\author{
Paulo A. Faria da Veiga and Michael O'Carroll \\ Departamento de Matemática, ICMC-USP, C.P. 668, 13560-970 São Carlos SP, Brazil
}

January 15, 2002.

\begin{abstract}
We determine the effects of nonlocal, nonlinear interactions on the excitation spectrum of lattice quantum field scalar models. We consider perturbations of a quantized discrete string formally self-adjoint Hamiltonian operator on the lattice $\mathbb{Z}^{d}$, and with a large mass coefficient for the quadratic term. The low-lying energy-momentum spectrum has an isolated dispersion curve and a two-particle (first) band. We analyze a ladder approximation of the Bethe-Salpeter equation on the lattice, for a weak perturbation of the type $\sum_{\vec{x} \in \mathbb{Z}^{d}}\left[\lambda_{6}: \varphi(\vec{x})^{6}:+V(\varphi(\vec{x})], \lambda_{6}>0\right.$, and consider the spectral interval starting at zero and extending to near the three-particle threshold. For space dimension $d=1,2$ and $V(\varphi(\vec{x}))=\lambda_{1}: \varphi(\vec{x})^{4}:$, we find that a bound state occurs either below (if $\lambda_{1}<0$ ) or above the first band (if $\lambda_{1}>0$ ), but not both. This agrees with recent results where bound states were obtained for the stochastic dynamics generator associated with the relaxation rate to equilibrium in weakly coupled stochastic Ginzburg-Landau models with continuous time and on a spatial lattice $\mathbb{Z}^{d}$. These results are in contrast, however, with those obtained for $V(\varphi(\vec{x}))=\lambda_{2}: \varphi(\vec{x})^{3}(-\Delta \varphi)(\vec{x}):$. For this case, we show that stable particles exist above and below the band, for $d=1,2$, regardless the sign of the coupling $\lambda_{2}$. If $V(\varphi(\vec{x}))=\lambda_{3}: \varphi(\vec{x})^{2}\left(-\Delta \varphi^{2}\right)(\vec{x}):$, the ladder analysis is inconclusive. If $d=3, \ldots$, no bound states exist in the spectral region we consider.
\end{abstract}

PACS number(s): 02.50.-r, 02.30.Tb, 05.50.+q, 11.10.St, 02.30.Tb

KEY WORDS: Spectral Analysis, Bethe-Salpeter Equation, Bound States, Nonlocal Interactions, Scalar Models, Stochastic Dynamics, Ginzburg-Landau Models.

Submitted to Journal of Physics A.

Short running title: Nonlocal Interactions and Spectrum

E-mail addresses: veiga@icmc.sc.usp.br and ocarroll@icmc.sc.usp.br.

*Partially supported by Pronex, CNPq (Brazil) and FAPESP. 


\section{Introduction}

The analysis of systems governed by stochastic dynamics is of physical and purely mathematical interest. In physics, these systems are worth studying for several reasons e.g. because some classes of stochastic models can be used to describe the evolution of an order parameter in statistical mechanical systems [1,2]. In these cases, as a consequence of the spectral theorem, determining the excitation spectrum of the stochastic dynamics generator gives information about the relaxation rate to equilibrium.

Recently, much attention has been devoted to understand the quasi-particle or spectral structure of the stochastic dynamics generator in lattice models $[3,4,5,6,7]$. In these works, the picture of a massive particle and a bound state below the two-particle threshold (the bottom of the first, two-particle, band) is established, depending on the space dimension $d$, and on specific conditions on the interaction. Later, concentrating on weakly coupled stochastic Ginzburg-Landau (G-L) models defined on the spatial lattice $\mathbb{Z}^{d}$ and with continuous time, the work of $[6,7]$ was furthered by [8] where it is established, in the ladder approximation, that a stable particle may also exist above the top of the first band, with a concave dispersion for small momentum, for $d=1,2$. This is in contrast with the previous works where the dispersion is convex for the stable particle below the band, when it is present.

The analysis in $[6,7,8]$ concentrates on the spectrum for the stochastic dynamics generator in an interval that starts at zero and goes up to near the three-particle threshold. It is based on solving the Bethe-Salpeter (BS) equation, after applying a Feynman-Kac formula to map the problem into the problem of analyzing correlations of an associated massive imaginary-time scalar quantum field theory on the spatial lattice.

Regarding the spectral (bound state) analysis up to near the three-particle threshold, there are four main points that make the stochastic G-L models different from relativistic Euclidean quantum field theories that have been studied long ago in e.g. $[9,10,11]$ (see also [12]). First, because of the underlying stochastic character of the problem [see (1)] with the G-L action [see (2)], besides the usual local interactions, the associated scalar quantum field theory also presents mildly nonlocal interactions of the type $\lambda: \mathcal{P}^{\prime}(\varphi)\left(-\Delta+m^{2} / 2\right) \varphi$ : where $\lambda$ is the coupling constant, $\mathcal{P}(\varphi)$ an even polynomial starting with $\varphi^{4},-\Delta$ is the $\mathbb{Z}^{d}$-lattice Laplacian, $m$ a mass parameter which is taken large to ensure the existence of the infinite-lattice dynamic correlation functions, and : : denotes a Wick ordering (see below). Secondly, the B-S kernel corresponds to a rank-two operator on a convenient space while for an Euclidean local quantum field theory the rank is one. Thirdly, it is appropriate to observe that the use of relative "mixed coordinates" is a technical point that plays an important role in the whole analysis of the stochastic lattice G-L model. Replacing the commonly used relative and center of mass coordinates for the models on the continuum, they are given by a relative center of mass time coordinate and by relative coordinates for the space lattice. Finally, we remark that the first band in massive field theories on the continuum extends to infinity since for a system with two identical particles and with fixed center of mass momentum $\vec{p}=\vec{p}_{1}+\vec{p}_{2}$ the energy varies from $2\left[\vec{p}^{2} / 4+m^{2}\right]^{1 / 2}$ to infinity where $m$ is the rest mass of each particle. Thus, there is no band structure like the one found in $[6,7,8]$ for the lattice stochastic model.

Some natural questions arise from this analysis. First, one should try to find a good description of the physical properties of the particle described in [8]. Second, one should try to understand better by what mechanism they can arise in stochastic models. Third, since the rank of the B-S operator is two, one could expect to have two stable particles existing simultaneously. One should then try to find out whether or not this occurs, and give conditions for the two spectral points to coexist.

This paper addresses these questions. In section 2, we make our motivation more concrete and establish the framework and tools used for the bound state analysis in this paper, by describing the lattice stochastic G-L models and summarizing the results of $[6,7,8]$.

We go beyond the analysis of $[6,7,8]$ and show that the lowest power nonlocal interaction $\lambda: \varphi^{3}(-\Delta+$ $\left.m^{2} / 2\right) \varphi$ : gives rise to a rank-two B-S operator. The two bound states, above and below the two-particle band, as described in [8] exist only if the space dimension is $d=1,2$, and under a complementary assumption on the interaction. This assumption is related to the sign of the quartic interacting term [see (3)], which is governed by a coefficient denoted by $a_{2}$. For $d=1,2$ the bound state below the band exists for $a_{2}<0$ while the one above the band exists for $a_{2}>0$. Since the value of the free single mass $m^{2} / 2$ is taken large, and momenta are bounded on the infinite lattice, with values in $[-\pi, \pi]$, for both cases the nonlocal term $\lambda: \varphi^{3}(-\Delta) \varphi:$ is overshadowed by the local $\lambda m^{2} / 2: \varphi^{4}$ : term.

In section 3, this point is made clear by solving the ladder approximation of the B-S equation for a lattice 
$\lambda_{1}: \varphi^{4}$ : local scalar model associated with a discrete nonlinear quantized vibrating string. The ladder approximation consists in keeping only order $\lambda_{1}$ terms in the B-S kernel. Here, the rank of the B-S operator is one and for small $\lambda_{1}$, depending on its sign, we find a bound state above the first band $\lambda_{1}>0$ ) or a bound state below $\left.\lambda_{1}<0\right)$, but not both.

Next, we consider some simple nonlinear, nonlocal interactions which are nearest-neighbor. In the derivation of the stochastic dynamics generator for the lattice G-L model, local and nonlocal terms are present together and are constrained, having fixed relations among each other. In order to simplify our study, we do not take a more complicated combination of interaction terms, as occurs in the stochastic lattice G-L case. We thus consider separately several nonlocal interactions, so that we can understand the effect of each of them on stable state production. Higher power terms, which do not contribute to the B-S equation to the order we consider are added to the interaction in order to have local stability of the interacting potential. It is important to stress, however, that our analysis can be extended to other finite-range or suitably decaying infinite range nonlocalities, and more general nonlinearities. Also, throughout this paper, we use the ladder approximation, for large $m$ and for small $\lambda$.

In all cases, if $d \geq 3$, bound states below or above the band, up to near the three-particle threshold, do not occur. On the other hand, for $d=1,2$, it is shown that a nonlocal term of the type $\lambda_{2}: \varphi^{3}(-\Delta) \varphi:$, if present alone and for any sign of $\lambda_{2}$, indeed gives rise to two coexisting bound states near the two-particle band, one below and the other above the band. If a symmetrical term $\lambda_{3}: \varphi^{2}(-\Delta) \varphi^{2}:$ is considered, the ladder approximation is inconclusive and higher order terms in $\lambda_{3}$ have to be taken into account.

We make some concluding remarks in section 4 . We point out that the questions that are considered in this paper also arise in other contexts, for example, in the large time asymptotics of solutions of nonlinear classical wave equations. There is also the question of the existence of soliton and soliton-antisoliton type solutions when nonlocal nonlinearities occur. Nonlocal, nonlinear interactions are also present in quantum field theories when there is more than one field and functional integration over one of them is performed. This happens, for example, in Yukawa, QED, QCD or the Gross-Neveu model with an ultralocal field.

\section{The Lattice Stochastic G-L Model}

In this section, we establish the framework for the bound state analysis for lattice models. We define the lattice stochastic G-L systems, review the results of $[6,7,8]$ and define the main tools that will be used in the rest of the paper. These systems are given by the following Langevin type equation

$$
\frac{\partial \varphi}{\partial t}(t, \vec{x})=-\frac{1}{2} \frac{\delta}{\delta \varphi(t, \vec{x})} A(\varphi(t, \vec{x}))+\eta(t, \vec{x}) \quad ; \quad \varphi(\vec{x}, 0)=\psi(\vec{x}),
$$

where, for space coordinates $\vec{x} \in \mathbb{Z}^{d}$ and time $t \in \mathbb{R}, \varphi(\vec{x})$ is a set of unbounded real continuous scalar spin variables , $\psi(\vec{x})$ is some initial condition, $A$ is the system action and $\{\eta(t, \vec{x})\}, \vec{x} \in \mathbb{Z}^{d}, t \in[0, \infty)$ is a family of Gaussian white noise processes with expectations $E(\eta(t, \vec{x}))=0$ and $E\left(\eta(t, \vec{x}) \eta\left(t^{\prime}, \vec{y}\right)\right)=\delta\left(t-t^{\prime}\right) \delta_{\vec{x}, \vec{y}}$. The action is of G-L type, i.e.

$$
A(\varphi(\vec{x}))=\sum_{\vec{x} \in \mathbb{Z}^{d}}\left\{\frac{1}{2}\left[\sum_{i=1}^{d}\left[\varphi\left(\vec{x}+\vec{e}_{i}\right)-\varphi(\vec{x})\right]^{2}+m^{2} \varphi(\vec{x})^{2}\right]+\lambda \mathcal{P}(\varphi(\vec{x}))\right\} .
$$

Here, $\vec{e}_{i}$ is the unit vector along the $i$ th coordinate; $m>0$ and $\lambda \geq 0$ and $\mathcal{P}$ is an even polynomial of degree $2 N$, bounded from below and starting with a quartic term, given by

$$
\mathcal{P}(\varphi)=\sum_{n=2}^{N} \frac{a_{n}}{(2 n) !}: \varphi^{2 n}:
$$

with $a_{N}>0$ and : $\quad$ : meaning Wick order with respect to the covariance

$$
C=\left[-\frac{d^{2}}{d t^{2}}+\left(-\Delta+m^{2}\right)^{2}\right]^{-1}
$$


where $-\Delta$ is the lattice Laplacian $(-\Delta \varphi)(\vec{x})=2 d \varphi(\vec{x})-\sum_{|\vec{x}-\vec{y}|=1} \varphi(\vec{y})$.

The dynamics introduced by (1) for the Markov process $\varphi(t)=\varphi(t, \vec{x})$ associates a Markov semi-group and leaves invariant the Gibbs probability distribution $d \mu=e^{-A(\varphi)} d \varphi /$ normalization defined by the action (2). The time evolution of any function $f$ of the spin configuration $\varphi(\vec{x})$ is given by $f_{t}(\psi)=E(f(\varphi(t)))$, with $\varphi(t=0)=\psi(\vec{x})$. It follows that $f_{t}$ is determined by the Markov semi-group $\exp (-t B)$ with generator $B$, for $f=f(\{\varphi(\vec{x})\})$, given by

$$
B f=-\frac{1}{2} \sum_{\vec{x} \in \mathbb{Z}^{d}}\left[\frac{\delta^{2}}{\delta \varphi(\vec{x})^{2}} f-\frac{\delta A}{\delta \varphi(\vec{x})} \frac{\delta f}{\delta \varphi(\vec{x})}\right] .
$$

The spectrum of $B$ is related to decay rates of correlation functions of an imaginary continuous time space lattice quantum field theory through a Feynman-Kac formula (see $[6,7,8])$. Rather than analyze the spectrum of $B$, we consider the unitarily equivalent Schrödinger Hamiltonian-like operator

$$
\begin{aligned}
H= & -\frac{1}{2} \sum_{\vec{x} \in \mathbb{Z}^{d}} \frac{\delta^{2}}{\delta \varphi(\vec{x})^{2}}+\frac{1}{4} \sum_{\vec{x} \in \mathbb{Z}^{d}}\left[\frac{1}{2}\left(\frac{\delta A}{\delta \varphi(\vec{x})}\right)^{2}-\frac{\delta^{2} A}{\delta \varphi(\vec{x})^{2}}\right] \\
= & -\frac{1}{2} \sum_{\vec{x} \in \mathbb{Z}^{d}} \frac{\delta^{2}}{\delta \varphi(\vec{x})^{2}}+\frac{1}{8} \sum_{\vec{x} \in \mathbb{Z}^{d}} \varphi(\vec{x})\left[\left(-\Delta+m^{2}\right)^{2} \varphi\right](\vec{x}) \\
& +\frac{\lambda}{4} \sum_{\vec{x} \in \mathbb{Z}^{d}}\left[\left(-\Delta+m^{2}\right) \varphi\right](\vec{x}) \mathcal{P}^{\prime}(\varphi(\vec{x}))+\sum_{\vec{x} \in \mathbb{Z}^{d}}\left[\frac{\lambda^{2}}{8} \mathcal{P}^{\prime}(\varphi(\vec{x}))^{2}-\frac{\lambda}{4} \mathcal{P}^{\prime \prime}(\varphi(\vec{x}))-\frac{\left(2 d+m^{2}\right)}{4}\right] .
\end{aligned}
$$

The lattice translation operator $T(\vec{x})=\exp [-i \vec{P} \cdot \vec{x}]$ commutes with $H ; H$ is additively normalized so that its spectrum is positive and starts at zero. The joint spectrum of $H, \vec{P}$, where $\vec{P}$ is the self-adjoint momentum operator, is then analyzed.

The above infinite-lattice formulas are formal. In [7], it was rigorously shown how to define them starting from the finite-lattice finite-time models and then taking the thermodynamic limit using a cluster expansion, assuming $m$ large and $\lambda$ sufficiently small.

If $\lambda=0$, the spectrum can be determined exactly; the associated correlation functions are moments of a Gaussian measure with covariance $C$ given in (4). There is a natural quasi-particle interpretation for the $H, \vec{P}$ spectrum. Spectral points are denoted by $(E, \vec{p}), E \geq 0$ and $\vec{p} \in \mathbf{T}^{d} \equiv[-\pi, \pi]^{d}$. The point $(E, \overrightarrow{0})$ is referred to as a mass and $(0, \overrightarrow{0})$ is the vacuum. Moreover, we have a quasi-particle energy-momentum $(e-m)$ spectrum $\left(E_{0}(\vec{p}) \equiv E_{\lambda=0}(\vec{p}), \vec{p}\right)$, where $E_{0}(\vec{p})$ is the isolated dispersion curve

$$
E_{0}(\vec{p})=\sum_{i=1}^{d}\left(1-\cos p^{i}\right)+m^{2} / 2 \quad, \quad \vec{p}=\left(p^{1}, \ldots, p^{d}\right),
$$

with mass $m^{2} / 2$. The rest of the spectrum is given by $\cup_{\vec{p} \in \mathbf{T}^{d}} \cup_{n \geq 2}\left(E_{0, n}(\vec{p}), \vec{p}\right)$ with $E_{0, n}(\vec{p})=\sum_{j=1}^{n} E_{0}\left(\vec{p}_{j}\right)$, $\sum_{j=1}^{n} \vec{p}_{j}=\vec{p}$, i.e. the $e-m$ spectrum of $n$ non-interacting quasi-particles with total momentum $\vec{p}$. As the system is defined on a spatial lattice, the corresponding spectral regions may correspond to spectral bands. When this is the case, they can be determined by applying e.g the Lagrange multiplier method to the function $\sum_{j=1}^{n} E_{0}\left(\vec{p}_{j}\right), \sum_{j=1}^{n} \vec{p}_{j}=\vec{p}$, to compute the upper and lower envelopes. For instance, for $n=2$ and $d=1$, the band has lower envelope $E_{0,2}^{\downarrow}(\vec{p})=4 \sin ^{2}(p / 4)+m^{2}$, and upper envelope $E_{0,2}^{\uparrow}(\vec{p})=4 \cos ^{2}(p / 4)+m^{2}$. For sufficiently large $m$ and $n \geq 3$, any $d$, there is also band spectrum but, for sufficiently large $n$, the bands overlap. We are considering the large $m$ case so that there is at least a first isolated band.

The results of $[6,7,8]$ concern the weakly interacting case $0<\lambda<<1$; first, within the ladder approximation in [6] and then analyzing the complete model in [7], controlling perturbations about the ladder case. If $m$ is fixed large and $\lambda$ chosen sufficiently small, it is shown that a quasi-particle persists with dispersion curve $E_{\lambda}(\vec{p}) \geq E_{\lambda}(\overrightarrow{0})=m^{2} / 2+\mathcal{O}\left(\lambda^{2}\right)$. The mass spectrum up to the two-particle threshold mass $2 E_{\lambda}(\overrightarrow{0})$ is also determined. More precisely, for $d=1,2$ and if $a_{2}<0$, there is a single point $M_{b}$ in the mass spectrum interval $I_{\lambda}=\left(E_{\lambda}(\overrightarrow{0}), 2 E_{\lambda}(\overrightarrow{0})\right)$, located near $2 E_{\lambda}(\overrightarrow{0})$. The bound state is absent and there is no mass spectrum in $I_{\lambda}$ if $a_{2}>0$, for any $d$, or for $a_{2}<0$ and $d \geq 3$. In [8], the mass spectrum is considered for the interband region between the first (with envelopes $E_{\lambda, 2}^{\downarrow}(\vec{p})$ and $E_{\lambda, 2}^{\uparrow}(\vec{p})$ ) and the second (with envelopes $E_{\lambda, 3}^{\downarrow}(\vec{p})$ and $E_{\lambda, 3}^{\uparrow}(\vec{p})$ ) bands which, because of the weak interaction, are shown to be small deformations of the $\lambda=0$ bands. In the ladder approximation, and for $a_{2}>0$ and $d=1,2$, it is shown that there is mass spectrum $M_{a}$ above and 
close to the mass of the upper envelope of the first band. Furthermore, for small $|\vec{p}|$, it is shown in [8] that the associated dispersion curve for this interband state is concave while that of the bound state below the first band is convex. The results are depicted in Figure 1.

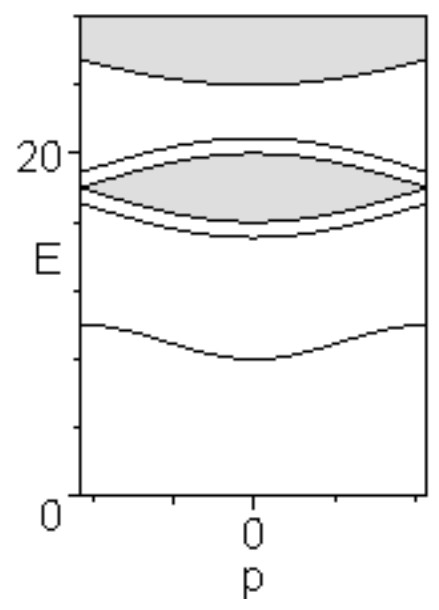

Figure 1: The approximate $e-m$ spectrum for the case $d=1$ and $m^{2}=16$. For $a_{2}<0$, only the isolated bound state lower dispersion curve appears; for $a_{2}>0$, only the isolated upper curve appears.

Our method is related to the method of $[13,14]$ used in the analysis of the continuum models. As in $[13,14]$, an associated imaginary continuous time, space lattice quantum field theory is constructed using standard methods furnishing a Hilbert space $\mathcal{H}$, commuting self-adjoint $e-m$ operators, time zero field operators and a vacuum vector. Vacuum expectation values of products of imaginary-time Heisenberg field operators are related to correlation functions by the Feynman-Kac formula, for $x_{1} \equiv\left(t_{1}, \vec{x}_{1}\right), \ldots$ and $t_{1} \leq t_{2} \leq t_{3} \ldots \leq t_{n}$,

$$
\begin{aligned}
S_{\lambda}\left(x_{1} ; \ldots ; x_{n}\right) & \equiv\left\langle\varphi\left(x_{1}\right) \ldots \varphi\left(x_{n}\right)\right\rangle \\
& =\left(\Omega, \hat{\phi} e^{-\left(t_{2}-t_{1}\right) H+i \vec{P} \cdot\left(\vec{x}_{2}-\vec{x}_{1}\right)} \hat{\phi} \ldots e^{-\left(t_{n}-t_{n-1}\right) H+i \vec{P}_{\cdot} \cdot\left(\vec{x}_{n}-\vec{x}_{n-1}\right)} \hat{\phi} \Omega\right),
\end{aligned}
$$

where $\langle\cdot\rangle$ corresponds to the average given by a functional integral with a path space measure $d \rho=\frac{e^{-W} d \nu}{\int e^{-W} d \nu}$, with $W \equiv W(\varphi)$ given by

$$
W=\int d t \sum_{\vec{x} \in \mathbb{Z}^{d}}\left[\frac{\lambda}{4} \mathcal{P}^{\prime}(\varphi(\vec{x}, t))\left(-\Delta+m^{2}\right) \varphi(\vec{x}, t)+\frac{\lambda^{2}}{8} \mathcal{P}^{\prime}(\varphi(\vec{x}, t))^{2}-\frac{\lambda}{4} \mathcal{P}^{\prime \prime}(\varphi(\vec{x}, t))\right],
$$

and $d \nu$ is a normalized Gaussian measure with mean zero and covariance $C$ of (4) with kernel

$$
C(x, y)=C\left(t, \vec{x} ; t^{\prime}, \vec{y}\right)=\int \varphi(t, \vec{x}) \varphi\left(t^{\prime}, \vec{y}\right) d \nu=\frac{1}{(2 \pi)^{d+1}} \int_{-\infty}^{\infty} d p_{0} \int_{\mathbf{T}^{d}} d \vec{p} \frac{e^{i p_{0}\left(t-t^{\prime}\right)} e^{i \vec{p} \cdot(\vec{x}-\vec{y})}}{p_{0}^{2}+\left[\frac{\left(\vec{p}_{\ell}\right)^{2}}{2}+\frac{m^{2}}{2}\right]^{2}}
$$

where $\left(\vec{p}_{\ell}\right)^{2} \equiv 2 \sum_{i=1}^{d}\left(1-\cos p^{i}\right), \vec{p}=\left(p^{1}, \ldots, p^{d}\right) \in \mathbf{T}^{d}$ and $\vec{p} \cdot(\vec{x}-\vec{y})=\sum_{i=1}^{d} p^{i}\left(x^{i}-y^{i}\right)$.

The above infinite-lattice infinite-time formulas for correlation functions (see [7]) are controlled by cluster and Mayer expansions, and incorporate the time hyperplane decoupling expansion developed in [9] that allows us to prove that certain truncated two and four-point functions have tree exponential decay with exponents showing the correct two and three-particle irreducible behavior. We observe that these space-time decay properties imply analyticity properties in the Fourier space which are used to obtain the spectral results.

In order to analyze the two-particle spectrum, we consider the Fourier transform of a partially truncated four-point correlation function expressed in terms of mixed relative coordinates (relative temporal center of 
mass coordinates and relative spatial coordinates) $\tilde{D}_{\lambda}(p, q, k)$, where $k=\left(k^{0}, \vec{k}\right), k^{0} \in \mathbb{R}, \vec{k} \in \mathbf{T}^{d} . D_{\lambda}$ is the solution of the B-S equation

$$
D_{\lambda}=D_{\lambda}^{0}+D_{\lambda} K_{\lambda} D_{\lambda}^{0}
$$

where, letting $S_{\lambda}$ denote the two-point function for the interacting model, $D_{\lambda}^{0}\left(x_{1}, x_{2}, x_{3}, x_{4}\right)=S_{\lambda}\left(x_{1}, x_{3}\right)$ $S_{\lambda}\left(x_{2}, x_{4}\right)+S_{\lambda}\left(x_{1}, x_{4}\right) S_{\lambda}\left(x_{2}, x_{3}\right)$ and $D_{\lambda}\left(x_{1}, x_{2}, x_{3}, x_{4}\right)=S_{\lambda}\left(x_{1}, x_{2}, x_{3}, x_{4}\right)-S_{\lambda}\left(x_{1}, x_{2}\right) S_{\lambda}\left(x_{3}, x_{4}\right)$, and $S_{\lambda}\left(x_{1}, x_{2}, x_{3}, x_{4}\right)$ is the four-point function.

The mixed relative coordinates are given by

$$
\xi=\left(x_{2}^{0}-x_{1}^{0}, \vec{x}_{2}-\vec{x}_{1}\right) ; \eta=\left(x_{4}^{0}-x_{3}^{0}, \vec{x}_{4}-\vec{x}_{3}\right) ; \tau=\left[\frac{1}{2}\left(x_{3}^{0}+x_{4}^{0}-x_{1}^{0}-x_{2}^{0}\right), \vec{x}_{3}-\vec{x}_{2}\right] .
$$

By translational invariance, $D_{\lambda}\left(x_{1}, x_{2}, x_{3}, x_{4}\right)=D_{\lambda}\left(0, x_{2}-x_{1}, x_{3}-x_{1}, x_{4}-x_{1}\right)$ and, by abuse of notation, we write the r.h.s. as $D_{\lambda}(\xi, \eta, \tau)$ after expressing it in terms of the mixed relative coordinates. We proceed similarly for $D_{\lambda}^{0}$ and $K_{\lambda}$.

Using the symmetries of $D_{\lambda}$ and $D_{\lambda}^{0}$ (hence also of $K_{\lambda}$ ), namely $x_{1} \leftrightarrow x_{2}, x_{3} \leftrightarrow x_{4}, x_{1}, x_{2} \leftrightarrow x_{3}, x_{4}$, $x_{1}, x_{2}, x_{3}, x_{4} \leftrightarrow-x_{1},-x_{2},-x_{3},-x_{4}$, the B-S equation for $\tilde{D}(k)$ is

$$
\tilde{D}_{\lambda}(k)=\tilde{D}_{\lambda}^{0}(k)+(2 \pi)^{-2(d+1)} \tilde{D}_{\lambda}(k) \tilde{K}_{\lambda}(k) \tilde{D}_{\lambda}^{0}(k)
$$

where e.g. $\tilde{D}_{\lambda}(k)$ is defined by the kernel $\tilde{D}_{\lambda}(p, q, k)$ through

$$
\left(\tilde{D}_{\lambda}(k) f\right)(p)=\int \tilde{D}_{\lambda}(p, q, k) f(q) d q
$$

and $(p, q, k)$ are the momentum variables conjugate to $(\xi, \eta, \tau)$. Similar expressions hold for $\tilde{D}_{\lambda}^{0}$ and $\tilde{K}_{\lambda}$.

As in works involving relativistic quantum field theories in the continuum $[9,10,11]$, recall from $[6,7,8]$ that the $e-m$ spectrum associated with the two-particle states occurs as a $k^{0}$ singularity in the positive imaginary axis of the Fourier transform of $D_{\lambda}$. This is so because, for any function $f: \mathbb{Z}^{d} \rightarrow \mathbb{C}$, vanishing outside a finite set, a calculation yields

$$
\begin{aligned}
\left(f, D_{\lambda} f\right)(k) & =\int d p \int d q \tilde{f}^{*}(\vec{p}) \tilde{D}_{\lambda}(p, q, k) \tilde{f}(\vec{q}) \\
& =\int_{0}^{\infty} \int_{\mathbf{T}^{d}} \frac{2 E}{\left(k^{0}\right)^{2}+E^{2}}(2 \pi)^{3 d+2} \delta(\vec{q}-\vec{k}) d(\theta(f), \mathcal{E}(E, \vec{q}) \theta(f))
\end{aligned}
$$

where $\mathcal{E}(E, \vec{p})$ is the spectral family for $(H, \vec{P}), \theta(f)=\sum_{\vec{x} \in \mathbb{Z}^{d}} f(\vec{x}) \theta(-\vec{x})$, with $\theta(\vec{\eta})=\hat{\varphi}(\overrightarrow{0}) \hat{\varphi}(\vec{\eta}) \Omega-(\Omega, \hat{\varphi}(\overrightarrow{0}) \hat{\varphi}(\vec{\eta}) \Omega) \Omega$.

The determination of the spectrum in $[6,7]$ above the one-particle state and below the two-particle threshold is restricted to total momentum $\vec{k}=\overrightarrow{0}$, i.e. only to mass spectrum. We write $K_{\lambda}=\left(D_{\lambda}^{0}\right)^{-1}-\left(D_{\lambda}\right)^{-1}$ as $K_{\lambda}=\lambda L+\lambda^{2} K_{\lambda}^{(2)}$, where $\lambda L$ is called the ladder approximation to $K_{\lambda}$. The ladder kernel is calculated to be $\tilde{L}(p, q, k)=-\frac{3}{4} a_{2}\left[E_{0}(\vec{p})+E_{0}(\vec{q})+E_{0}(\vec{p}-\vec{k})+E_{0}(\vec{q}-\vec{k})\right]$ and has rank two.

We now obtain an explicit solution for $\tilde{D}_{\lambda}(p, q, k)$ in the ladder approximation. Letting $S_{\lambda}$ denote the two-point function, the kernel of $\tilde{D}_{\lambda}^{0}$ is given by

$$
\begin{gathered}
\tilde{D}_{\lambda}^{0}(p, q, k)=(2 \pi)^{d+1} \delta\left(p^{0}+q^{0}\right)\left[\tilde{S}_{\lambda}\left(\frac{k^{0}}{2}-p^{0}, \vec{p}\right) \tilde{S}_{\lambda}\left(\frac{k^{0}}{2}+p^{0}, \vec{q}\right) \delta(\vec{p}+\vec{q}-\vec{k})\right. \\
\left.+\tilde{S}_{\lambda}\left(\frac{k^{0}}{2}+p^{0}, \vec{p}\right) \tilde{S}_{\lambda}\left(\frac{k^{0}}{2}-p^{0}, \vec{k}-\vec{p}\right) \delta(\vec{p}-\vec{q})\right] .
\end{gathered}
$$

Using one of the symmetry properties above, for $\vec{k}=\overrightarrow{0}$, we have $\tilde{D}_{\lambda}^{0}\left(p, q, k^{0}\right)=\tilde{D}_{\lambda}^{0}\left(-p, q, k^{0}\right)$, and similarly for $\tilde{D}_{\lambda}$ and $L$. For this reason, we consider the B-S equation only in the space of symmetric functions $f(p)=f(-p)$. Suppressing the $k^{0}$ dependence, it reads

$$
\tilde{D}_{\lambda}(p, q)=r_{0}(p) \delta(p+q)+\lambda^{\prime} r_{0}(q) E_{0}(\vec{q}) \int \tilde{D}_{\lambda}(p, u) d u+\lambda^{\prime} r_{0}(q) \int \tilde{D}_{\lambda}(p, u) E_{0}(\vec{u}) d u
$$


where $r_{0}(q) \equiv r_{\lambda, 0}=2 \tilde{S}_{\lambda}\left(\frac{k^{0}}{2}-q^{0}, \vec{q}\right) \tilde{S}_{\lambda}\left(\frac{k^{0}}{2}+q^{0}, \vec{q}\right)$. In (8), we set $\lambda^{\prime}=-3(2 \pi)^{2(d+1)} a_{2} \lambda / 2$ and we have used $\tilde{S}_{\lambda}\left(p^{0}, \vec{p}\right)=\tilde{S}_{\lambda}\left(p^{0},-\vec{p}\right)$.

Multiplying (8) successively by 1 and $E_{0}(\vec{q})$, integrating over $q$, and solving the two resulting equations for $X(p) \equiv \int \tilde{D}(p, u) d u$ and $Y(p) \equiv \int \tilde{D}(p, u) E_{0}(\vec{u}) d u$, we find

$$
\begin{aligned}
\tilde{D}(p, q)=r_{0}(p) \delta(p+q)-\lambda^{\prime}[\beta \gamma- & \left.(\alpha-1)^{2}\right]^{-1} r_{0}(p) r_{0}(q) \times \\
& {\left[\beta E_{0}(\vec{p}) E_{0}(\vec{q})+(1-\alpha)\left(E_{0}(\vec{p})+E_{0}(\vec{q})\right)-\gamma\right] }
\end{aligned}
$$

where

$$
\begin{aligned}
& \alpha \equiv \alpha_{\lambda}\left(k^{0}\right)=\lambda^{\prime} \int_{\mathbf{T}^{d}} E_{0}(\vec{q}) G\left(\vec{q}, k^{0}\right) d \vec{q}, \\
& \beta \equiv \beta_{\lambda}\left(k^{0}\right)=\lambda^{\prime} \int_{\mathbf{T}^{d}} G\left(\vec{q}, k^{0}\right) d \vec{q}, \\
& \gamma \equiv \gamma_{\lambda}\left(k^{0}\right)=\lambda^{\prime} \int_{\mathbf{T}^{d}} E_{0}(\vec{q})^{2} G\left(\vec{q}, k^{0}\right) d \vec{q} .
\end{aligned}
$$

where

$$
G\left(\vec{q}, k^{0}\right)=\int r_{0}(q) d q^{0}=2 \int \tilde{S}_{\lambda}\left(\frac{k^{0}}{2}+q^{0}, \vec{q}\right) \tilde{S}_{\lambda}\left(\frac{k^{0}}{2}-q^{0}, \vec{q}\right) d q^{0} .
$$

Now, recalling from $[6,7]$ that $k^{0}$ singularities of (6) are points in the $e-m$ spectrum, we see from (9) that we need to analyze the $k^{0}$ singularities of $\alpha, \beta$ and $\gamma$. From $[6,7], \tilde{S}_{\lambda}(p)$ has the representation

$$
\tilde{S}_{\lambda}(p)=\frac{c_{\lambda}(\vec{p})}{\left(p^{0}\right)^{2}+E_{\lambda}(\vec{p})^{2}}+\int_{m^{2}}^{\infty} \frac{2 E}{\left(p^{0}\right)^{2}+E^{2}} d \eta_{\lambda}^{\prime}(E, \vec{p})
$$

where $c_{\lambda}(\vec{p})=1+\mathcal{O}\left(\lambda^{2}\right)$ and $d \eta^{\prime}$ has support on odd states with more than one particle. Then, performing the $q^{0}$ integration in (10), we obtain

$$
G\left(\vec{p}, k^{0}\right)=2 \pi\left[c_{\lambda}(\vec{p})\right]^{2} \quad \frac{1}{E_{\lambda}(\vec{p})\left[\left(k^{0}\right)^{2}+\left[2 E_{\lambda}(\vec{p})\right]^{2}\right]}+G_{1}\left(\vec{p}, k^{0}\right) .
$$

where $G_{1}\left(\vec{p}, k^{0}\right)$ is analytic in $\operatorname{Im} k^{0} \in\left(0,3 m^{2} / 2\right)$. Also, from [7], the isolated one-particle dispersion curve $E_{\lambda}(\vec{p})$ behaves as $m^{2} / 2+a \sum_{j=1}^{d}\left(p^{j}\right)^{2}+\mathcal{O}\left(\lambda^{2}\right)$, with a constant $a>0$, for small $|\vec{p}|$. We thus see that the $k^{0}$ singularities of $\alpha, \beta$ and $\gamma$ lie in the first band.

From (9), we see that the only possible singularities in $\operatorname{Im} k^{0} \in\left(0,2 E_{\lambda}(\overrightarrow{0})\right)$ or above the first band up to near the three-particle threshold come from the zeroes of $\left[\beta \gamma-(\alpha-1)^{2}\right] \equiv\left(\mu_{+}-1\right)\left(\mu_{-}-1\right)$, for $\mu_{ \pm}\left(k^{0}\right)=$ $\lambda^{\prime}\left[\alpha\left(k^{0}\right) \pm\left(\beta\left(k^{0}\right) \gamma\left(k^{0}\right)\right)^{1 / 2}\right]$.

We now take $k^{0}$ on the positive imaginary axis and let it approach the lower envelope of the band from below ( $\alpha, \beta$ and $\gamma$ are positive) to obtain the bound state mass $M_{b}$ for $\mu_{+}(k)=1$, for $a_{2}<0$ and $d=1,2$. Letting it approach the upper envelope from above ( $\alpha, \beta$ and $\gamma$ are now negative), $\mu_{-}(k)=1$ gives us the mass $M_{a}$ for $a_{2}>0$ and $d=1,2$. For $d \geq 3$ there are no mass spectral points above or below the first band.

As estimate to the mass $M_{b}$ is given in [6, 7]. An estimate to the mass $M_{a}$ can be derived.

\section{$3 \quad$ Non-Locality Versus Locality}

To separate the effects of local and nonlocal, nonlinear terms on the spectrum of a lattice quantum field model, we consider perturbations of a quantized discrete string with a mass term, on the $\mathbb{Z}^{d}$ lattice, and continuous time. The Hamiltonian is

$$
H=-\frac{1}{2} \sum_{\vec{x} \in \mathbb{Z}^{d}} \frac{\delta^{2}}{\delta \varphi(\vec{x})^{2}}+\frac{1}{2} \sum_{\vec{x} \in \mathbb{Z}^{d}}\left[\varphi(\vec{x})\left(-\Delta+m^{2}\right) \varphi\right](\vec{x})+W(\varphi)
$$


where $W(\varphi)$ is an interaction potential.

The Hamiltonian (11) is the generator of a continuous-time semi-group and the correlation functions are obtained by a Feynman-Kac formula as in the previous section. With $W=0$, the correlation functions are Gaussian with covariance given now by $C=\left[-d / d t^{2}-\Delta+m^{2}\right]^{-1}$. The spectrum for the corresponding generator is as in the case of the lattice stochastic model but with the one-particle dispersion curve

$$
E_{0}(\vec{p})=\left[\sum_{j=1}^{d} 2\left(1-\cos p^{j}\right)+m^{2}\right]^{1 / 2}
$$

with mass $m>0$. As before, there is a band structure. For large $m$, the first band has a lower envelope given by $E_{0,2}^{\downarrow}(\vec{p})=2\left\{\sum_{j=1}^{d} 2\left[1-\cos \left(p^{j} / 2\right)\right]+m^{2}\right\}^{1 / 2}$, with $\vec{p}=\vec{p}_{1}+\vec{p}_{2}$ being the total spatial field momentum (note that each particle has momentum $\vec{p} / 2)$, and upper envelope $E_{0,2}^{\uparrow}(\vec{p})=2\left\{\sum_{j=1}^{d} 2\left[1+\cos \left(p^{j} / 2\right)\right]+m^{2}\right\}^{1 / 2}$.

Putting in $W(\varphi)$ a $\lambda_{6} \sum_{\vec{x}}: \varphi(\vec{x})^{6}:, \lambda_{6}>0$, term to ensure stability, we consider $W(\varphi)=V(\varphi)+\lambda_{6} \sum_{\vec{x}}$ : $\varphi(\vec{x})^{6}$ :, where $V(\varphi)$ is taken to be $V_{1}(\varphi)=\lambda_{1} \sum_{\vec{x}}: \varphi(\vec{x})^{4}:$ or $V_{2}(\varphi)=\lambda_{2} \sum_{\vec{x}}: \varphi(\vec{x})^{3}[-\Delta \varphi](\vec{x}):$ or $V_{3}(\varphi)=\lambda_{3} \sum_{\vec{x}}: \varphi(\vec{x})^{2}\left[-\Delta \varphi^{2}\right](\vec{x}):$

In any of the different models above, the two-point function and the one-particle dispersion curve obey similar properties as that of the stochastic model. In particular, we have $E_{\lambda}(\vec{p})=m^{2}+\mathcal{O}\left(\lambda^{2}\right)$, for $\lambda$ here being $\lambda_{1}$ or $\lambda_{2}$ or $\lambda_{3}$, depending on the case. Also, $2 E_{\lambda}(\vec{p})$ denotes the two-particle threshold, i.e. the beginning of the first band, etc.

The complete continuous model with $V_{1}$ and $d=1$ is studied in [11, 15]. A bound state is shown to exist below the two-particle threshold (beginning of the infinite band).

We now determine the mass spectrum for the cases $V_{1}, V_{2}$ and $V_{3}$ :

Case $V_{1}(\varphi)=\lambda_{1} \sum_{\vec{x}}: \varphi(\vec{x})^{4}:$

For the ladder approximation to $K_{\lambda}$, repeating the steps from the last section, suppressing the $\lambda$ sub-indices henceforth and up to unimportant positive multiplicative constants, we find

$$
L_{1}\left(x_{1}, x_{2}, x_{3}, x_{4}\right)=-\lambda_{1} \delta\left(x_{2}-x_{1}\right) \delta\left(x_{3}-x_{1}\right) \delta\left(x_{4}-x_{1}\right)
$$

so that in terms of the relative mixed coordinates (5)

$$
L_{1}(\xi, \eta, \tau)=-\lambda_{1} \delta(\xi) \delta(\eta) \delta(\tau) .
$$

Hence $\tilde{L}_{1}(p, q, k)=-\lambda_{1}$.

For $\vec{k}=\overrightarrow{0}$, the B-S equation for $\tilde{D}\left(p, q, k^{0}\right)$ becomes, omitting the $k^{0}$ dependence, and setting $\lambda_{1}^{\prime}=$ $(2 \pi)^{-2(d+1)} \lambda_{1}$,

$$
\tilde{D}(p, q)=r_{0}(p) \delta(p+q)-\lambda_{1}^{\prime} r_{0}(q) \int \tilde{D}(p, u) d u .
$$

Integrating over $q$, we solve for $\int \tilde{D}(p, u) d u$ so that

$$
\tilde{D}(p, q)=r_{0}(p) \delta(p+q)+r_{0}(p) \frac{-\lambda_{1}^{\prime}}{1+\lambda_{1}^{\prime} \int r_{0}(u) d u} r_{0}(q) .
$$

A possible mass point outside the band can occur when the denominator is zero, i.e. we have the condition

$$
-\lambda_{1}^{\prime} \int r_{0}(q) d q=1=-\lambda_{1}^{\prime} \int G\left(\vec{q}, k^{0}\right) d \vec{q} \equiv-\lambda_{1}^{\prime} I_{0}
$$

with $G\left(\vec{q}, k^{0}\right)$ given by $(10)$.

Taking into account the properties of the two-point function $\tilde{S}(q)$, and hence of $G\left(\vec{q}, k^{0}\right)$, we see that for all small $\left|\lambda_{1}\right|>0$ there is a bound state below the band for the case $\lambda_{1}<0$ and a bound state above the band for the case $\lambda_{1}>0$, if $d=1,2$. For $d \geq 3$, no state exists below or in an interval extending from above the band (if $\lambda_{1}>0$ ) to near the three particle threshold $3 E_{\lambda}(\vec{p})$. 
Case $V_{2}(\varphi)=\lambda_{2} \sum_{\vec{x}}: \varphi(\vec{x})^{3}[-\Delta \varphi](\vec{x}):$

Proceeding similarly, we find

$$
\begin{aligned}
L_{2}\left(x_{1}, x_{2}, x_{3}, x_{4}\right)=-\lambda_{2}\left[\delta\left(x_{3}-x_{1}\right)\right. & \delta\left(x_{4}-x_{1}\right)(-\Delta)\left(\vec{x}_{1}, \vec{x}_{2}\right) \delta\left(x_{1}^{0}-x_{2}^{0}\right) \\
+ & \left.\delta\left(x_{2}-x_{1}\right) \delta\left(x_{3}-x_{2}\right)(-\Delta)\left(\vec{x}_{4}, \vec{x}_{3}\right) \delta\left(x_{4}^{0}-x_{3}^{0}\right)\right]
\end{aligned}
$$

so that, after passing to relative coordinates, taking the Fourier transform and setting $\vec{k}=\overrightarrow{0}$, we obtain $\tilde{L}_{2}\left(p, q, k^{0}\right)=-\lambda_{2}[-\tilde{\Delta}(\vec{p})-\tilde{\Delta}(\vec{q})]$. The B-S equation and its solution have the form of equations $(7)$ and (8), with $E_{0}(\vec{p})$ replaced by $-\tilde{\Delta}(\vec{p})$. Thus, a mass point can occur if, with $\lambda_{2}^{\prime}=(2 \pi)^{-2(d+1)} \lambda_{2}$,

$$
\lambda_{2}^{\prime}\left[I_{1} \pm\left(I_{0} I_{2}\right)^{1 / 2}\right]=1 .
$$

with $I_{0}$ given in (12) and

$$
I_{1,2}=\int G\left(\vec{q}, k^{0}\right)[-\tilde{\Delta}(\vec{q})]^{1,2} d \vec{q}
$$

Approaching $2 E_{\lambda}(\vec{p})$ from below $\left(I_{0}, I_{1}\right.$ and $I_{2}$ are positive), for $d=1,2$ and if $\lambda_{2}>0$, there is a bound state, taking the plus sign in (13), since $I_{0}$ is singular. $I_{1}$ and $I_{2}$ are not singular. Approaching the band from above $\left(I_{0}, I_{1}\right.$ and $I_{2}$ are negative), there is also a state again taking the plus sign. Similarly, for $\lambda_{2}<0$, and taking the minus sign in (13), both states occur. However, for $d \geq 3$, there is no mass spectrum below or above the band.

Case $V_{3}(\varphi)=\lambda_{3} \sum_{\vec{x}}: \varphi(\vec{x})^{2}\left[-\Delta \varphi^{2}\right](\vec{x}):$

We find

$$
\begin{aligned}
L_{3}\left(x_{1}, x_{2}, x_{3}, x_{4}\right)= & -\lambda_{3}\left[\delta\left(x_{4}-x_{2}\right) \delta\left(x_{3}-x_{1}\right)(-\Delta)\left(\vec{x}_{1}, \vec{x}_{2}\right) \delta\left(x_{1}^{0}-x_{2}^{0}\right)\right. \\
& \left.+\delta\left(x_{3}-x_{2}\right) \delta\left(x_{4}-x_{1}\right)(-\Delta)\left(\vec{x}_{1}, \vec{x}_{2}\right) \delta\left(x_{1}^{0}-x_{2}^{0}\right)+\delta\left(x_{4}-x_{3}\right) \delta\left(x_{2}-x_{1}\right)(-\Delta)\left(\vec{x}_{1}, \vec{x}_{3}\right) \delta\left(x_{1}^{0}-x_{3}^{0}\right)\right]
\end{aligned}
$$

and thus, setting $\vec{k}=\overrightarrow{0}, \tilde{L}_{3}\left(p, q, k^{0}\right)=-\lambda_{3}[-\tilde{\Delta}(\vec{p}-\vec{q})-\tilde{\Delta}(\overrightarrow{0})]=-\lambda_{3}(-\tilde{\Delta})(\vec{p}-\vec{q})$ noting that $\tilde{\Delta}(\overrightarrow{0})=0$.

Now, consider $d=1$; the cases $d>1$ being similar. Since $(-\tilde{\Delta})(\vec{p}-\vec{u})=1-\cos (p-u)=1-$ $\cos p \cos u-\sin p \sin u$, the $\vec{k}=\overrightarrow{0}$ B-S equation for $\tilde{D}\left(p, q, k^{0}\right)$ becomes, with $\lambda_{3}^{\prime}=(2 \pi)^{-2(d+1)} \lambda_{3}$,

$$
\tilde{D}(p, q)=r_{0}(p) \delta(p+q)-\lambda_{3}^{\prime} r_{0}(q) \int \tilde{D}(p, u) d u+\lambda_{3}^{\prime} r_{0}(q) c(\vec{q}) \int \tilde{D}(p, u) c(\vec{u}) d u
$$

where $c(\vec{q})=\cos q^{1}$.

Multiplying successively equation (14) by 1 and $c(\vec{q})$, and integrating over $q$ gives two equations for $X(p) \equiv$ $\int \tilde{D}(p, u) d u$ and $Y(p) \equiv \int \tilde{D}(p, u) c(\vec{u}) d u$. Solving for $X(p)$ and $Y(p)$, we get

$$
\begin{array}{r}
\tilde{D}(p, q)=r_{0}(p) \delta(p+q)-\lambda_{3}^{\prime}\left[\left(1-I_{0}\right)\left(1+I_{2}\right)+I_{1}^{2}\right]^{-1} \times \\
r_{0}(p) r_{0}(q)\left[1+I_{2}-I_{1}(c(\vec{p})+c(\vec{q}))+\left(I_{0}-1\right) c(\vec{p}) c(\vec{q})\right]
\end{array}
$$

where

$$
I_{0,1,2}=-\lambda_{3}^{\prime} \int G\left(\vec{q}, k^{0}\right)[c(\vec{q})]^{0,1,2} d \vec{q} .
$$

Approaching the band from above or below, $I_{0}, I_{1}$ and $I_{2}$ as well as $I_{0} I_{2}-I_{1}^{2}$ become singular but $I_{0}-I_{2}$ does not. For $d=2$, similar behavior occurs. However, the determining equation is unreliable since we have kept only $\mathcal{O}\left(\lambda_{3}\right)$ terms in the B-S kernel, and the product of two I's is $\mathcal{O}\left(\lambda_{3}^{2}\right)$. Thus, we draw no conclusions about the existence or non-existence of mass spectrum, for the cases $d=1,2$. If $d \geq 3, I_{0}, I_{1}$ and $I_{2}$ are all finite, and there is no spectrum outside the band. 


\section{Conclusions}

We have determined the effect on the spectrum of a quantum $\mathbb{Z}^{d}$ lattice model Hamiltonian from zero up to near the three-particle threshold due to local and nonlocal, nonlinear interactions in a ladder approximation. In comparison with the case of the Hamiltonian associated with the Ginzburg-Landau stochastic model on the lattice treated previously in $[6,7,8]$, the qualitative effect of a local $\lambda_{1}: \varphi(\vec{x})^{4}:$ term is the same, namely it produces a bound state below the band for $\lambda_{1}<0$ and a state above the band for $\lambda_{1}>0$ in $d=1,2$. This behavior comes about as the nonlocal term in the Ginzburg-Landau Hamiltonian is $\lambda: \mathcal{P}^{\prime}(\varphi(\vec{x})(-\Delta \varphi)(\vec{x}):$, with mass $m^{2} / 2$ large, so the local part dominates and masks the effect of the nonlocality. On the other hand, the effect of a $\lambda_{2}: \varphi^{3}(\vec{x})(-\Delta \varphi)(\vec{x})$ : nonlocal nonlinearity produces a state both below and above the band for $d=1,2$, regardless of the sign of $\lambda_{2}$. In $d=1,2$, a higher order treatment is needed to see the effect of a $\lambda_{3}: \varphi^{2}(\vec{x})\left(-\Delta \varphi^{2}\right)(\vec{x}):$ term. For all the above perturbations, if $d \geq 3$, there are no states below the first band or above it up to near the three particle threshold. What about the effect of higher order (greater than four) nonlocal monomial Wick ordered interactions? In the ladder approximation, these do not contribute.

It would be interesting to determine nonlocal, nonlinear effects for classical wave equation large time asymptotics, their quantum versions, both in the scalar and multicomponent versions. Particularly, it is worthwhile to investigate the existence of degeneracies in the multicomponent case and the existence of soliton and solitonantisoliton solutions in the classical wave case.

\section{References}

[1] P. C. Hohenberg and B. I. Halperin, Theory of Dynamic Critical Phenomena, Rev. Mod. Phys. 49, 435-479 (1977).

[2] H. Spohn, Large Scale Dynamics of Interacting Particles, Springer Verlag, Berlin (1991).

[3] Yu. G. Kondratiev and R. A. Minlos, One-Particle Subspaces in the Stochastic XY Model, J. Stat. Phys. 87, 613-642 (1997).

[4] E. A. Zhizhina, Two-Particle Spectrum of the Generator for Stochastic Model of Planar Rotators at High Temperature, J. Stat. Phys. 91, 343-366 (1998).

[5] R. A. Minlos and Y. M. Suhov, On the Spectrum of the Generator of an Infinite System of Interacting Diffusions, Commun. Math. Phys. 206, 463-489 (1999).

[6] R. Schor, J. C. A. Barata, P. A. Faria da Veiga and E. Pereira, Spectral Properties of Weakly Coupled Landau-Ginzburg Stochastic Models, Phys. Rev. E59, Issue 3, 2689-2694 (1999).

[7] P. A. Faria da Veiga, M. O'Carroll, E. Pereira and R. Schor: Spectral Analysis of Weakly Coupled Stochastic Lattice Ginzburg-Landau Models, Commun. Math. Phys. 220, 377-402 (2001).

[8] P. A. Faria da Veiga, M. O'Carroll and R. Schor: Interband Spectrum of Weakly Coupled Stochastic Lattice Ginzburg-Landau Models, submitted to the Brief Reports section of Phys. Rev. E, September 12 (2001). Available from www.icmc.sc.usp.br/ veiga/briefrep.ps .

[9] T. Spencer: The Decay of the Bethe-Salpeter Kernel in $\mathcal{P}(\varphi)_{2}$ Quantum Field Models, Commun. Math. Phys. 44, 143-164 (1975).

[10] T. Spencer and F. Zirilli: Scattering States and Bound States in $\lambda \mathcal{P}(\phi)_{2}$ Models, Commun. Math. Phys. 49, 1-16 (1976).

[11] J. Dimock and J.-P. Eckmann: On the Bound State in Weakly Coupled $\lambda\left(\phi^{6}-\phi^{4}\right)_{2}$ Models, Commun. Math. Phys. 51, 41-54 (1976).

[12] J. Glimm and A. Jaffe: Quantum Physics: A Functional Integral Point of View, Springer Verlag, New York (1986). 
[13] J. Dimock: A Cluster Expansion for Stochastic Lattice Fields, J. Stat. Phys. 58, 1181-1207 (1990).

[14] G. Jona-Lasinio and R. Sénéor: Study of Stochastic Differential Equations by Constructive Methods. I., J. Stat. Phys. 83, 1109-1148 (1996).

[15] J. Dimock and J.-P. Eckmann: Spectral Properties and Bound State Scattering for Weakly Coupled $\mathcal{P}(\phi)_{2}$ Models, Annals of Physics 103, 289-314 (1977). 